ISSN-PRINT

1794-9831

E-ISSN 2322-7028

Vol. 15 No. 1

Ene - Jun 2018

Cúcuta, Colombia

Recibido:

23 Agosto

2017

Aprobado:

3 Octubre

2017

Autor de correspondencia

$\triangle$ Enfermera Magister. Docente Universidad del Cauca. Correo: adrianazuleta@ unicauca.edu.co. Popayán - Colombia.

${ }^{* *}$ Estudiantes X semestre Enfermería. Universidad del Cauca. Popayán, Colombia. Correo electrónico: jonfer@unicauca.edu. co, krojas@unicauca.edu.co, mltipan@unicauca.edu. co, carlostorresl@ unicauca.edu.co, linavallejo@unicauca.edu.co, luisfelipe@unicauca. edu.co

\section{Salud sexual reproductiva en estudiantes universitarios: conocimientos y prácticas}

\author{
Sexual and reproductive health in university students: beyond knowledge and \\ practices
}

\section{Saúde sexual reprodutiva em estudantes universitários: conhecimentos e práticas}

\author{
Ivett Adriana Herrera Zuleta* \\ Jonathan Fernando Reyes Salazar** \\ Katherine Patricia Rojas Velasco** \\ Magda Lizeth Tipán ${ }^{* *}$ \\ Carlos Andrés Torres López** \\ Lina Marcela Vallejo Trujillo** \\ Luis Felipe Zemanate Ordoñez ${ }^{* *}$
}

\section{Resumen}

Objetivo: Describir el conocimiento y las prácticas relacionadas con la salud sexual y reproductiva en los estudiantes de una Universidad del suroccidente colombiano. Materiales y métodos: Investigación cuantitativa, descriptiva y prospectiva de corte transversal realizada con estudiantes matriculados en I y II semestre en el primer periodo académico 2016. El cálculo de la muestra se obtuvo utilizando la fórmula de varianza desconocida y población total conocida (3.489), obteniendo un tamaño de muestra de 415 estudiantes. Se utilizó un instrumento tipo encuesta estructurado a partir del formato de consejería del programa de salud sexual y reproductiva universitario. Los datos se procesaron a través de Excel y el paquete estadístico SPSS versión 22. Para determinar la significación de la asociación se utilizó la prueba Chi cuadrado con una confiabilidad de $95 \%$. Resultados: En la población estudiada se encontró un predominio masculino del 50,7 \%, la edad promedio fue de 19 años, encontrando que el $81 \%$ está en una etapa de adolescencia tardía, el 62 $\%$ tiene un nivel de conocimientos deficientes sobre salud sexual y reproductiva, el $78 \%$ inició vida sexual con edad promedio de 16 años, un gran porcentaje de estos estudiantes tiene entre 1 y 3 compañeros(as) sexuales al año. El 70 \% utilizó condón en su primera relación sexual y el 12,3 \% de la población utilizó la píldora de emergencia. Conclusiones: El nivel de conocimiento acerca de salud sexual y reproductiva es deficiente. La mayoría de los estudiantes encuestados utiliza al menos un método de planificación familiar en sus relaciones sexuales.

Palabras clave: Sexualidad, Salud reproductiva, estudiantes, conocimientos y prácticas en salud.

\section{Abstract}

Objective: To describe the knowledge and practices related to Sexual and Reproductive Health in the students of a University of the Suroccidente Colombiano. Materials and methods: Quantitative descriptive prospective cross-sectional study; was carried out in students enrolled in I and II semester in the first academic period 2016; the calculation of the sample was obtained using the formula of

\section{Para citar este artículo/ To reference this article / Para citar este artigo/}

Herrera Zuleta IA, Reyes Salazar JF, Rojas Velasco KP, Tipán ML, Torres López CA, Vallejo Trujillo LM, Zemanate Ordoñez LF. Salud sexual reproductiva en estudiantes universitarios: conocimientos y prácticas. Rev. cienc. cuidad. 2018;15(1):58-70

Este es un artículo bajo la licencia CC BY (https://creativecommons.org/licenses/by/4.0/) @ @ 
unknown variance and known total population $(3,489)$, obtaining a sample size of 415 students. A structured survey-type instrument was developed based on the counseling format of the university's sexual and reproductive health program. Data were processed through Excel and the SPSS version 22 statistical package, to determine the significance of the association was used Chi square test with a reliability of $95 \%$. Results: A male prevalence of $50.7 \%$ was found in the study population, the mean age was 19 years, and $81 \%$ were in the late teens, $62 \%$ had a poor level of knowledge about sexual health and reproductive, $78 \%$ start sexual life with average age of 16 years, a large percentage of these students have between 1 and 3 sexual partners a year. Seventy percent used a condom at their first sexual intercourse and $12.3 \%$ of the population used the emergency pill. Conclusions: The level of knowledge about sexual and reproductive health is deficient. Most students surveyed use at least one method of family planning in their sexual relationships.

Keywords: Managing medication therapy, diagnosis, nurses, pediatrics.

\section{Sumario}

Objetivos: Descrever os conhecimentos e práticas relacionados com a saúde sexual e reprodutiva em estudantes de uma Universidado sudoeste Colombiano. Materiais e Métodos: A pesquisa quantitativa transversal descritiva prospectiva; foi realizado em estudantes matriculados em I e II, semestre 2016; cálculo da amostra foi obtida utilizando a fórmula de variância desconhecida e a população total conhecido (3489), obtendo-se um tamanho de amostra de 415 estudantes. Foi utilizado um tipo de instrumento de pesquisa estruturada. Os dados foram processados pelo pacote estatístico Excel versão 2010 e SPSS versão 22, para determinar o significado do teste foram utilizados do qui-quadrado de associação com uma confiabilidade de 95\%. Resultados: Na população estudada predominância do sexo masculino de $50,7 \%$, a idade média dos alunos era de 19 anos, descobrindo que $81 \%$ estão em uma fase de adolescência tardia, $62 \%$ têm um nível de conhecimentos regulares saúde sexual e reprodutiva, 78\% inicio vida sexual com uma idade média de 16 anos, uma grande porcentagem desses estudantes estão entre 1 e 3 parceiro (s) ano sexual. $70 \%$ usam preservativo em sua primeira relação sexual e $12,3 \%$ da população usava a pílula de emergência. Conclusões: O nível de conhecimento sobre a saúde sexual e reprodutiva é regular. A maioria dos estudantes pesquisados usar pelo menos um método de planejamento familiar em suas relações sexuais.

Palavras-chave: Gestão de terapia de medicação, diagnóstico, enfermeiros, pediatria.

\section{Introducción}

La salud sexual y reproductiva se define como "el bienestar físico, mental y social en el plano sexual y reproductivo" (1) y actualmente es un tema de gran importancia, ya que se considera como una problemática a nivel mundial, principalmente entre los jóvenes, quienes no han alcanzado una madurez emocional que les permita tener unas prácticas sexuales más seguras.

Se reconoce que para el año 2000, alrededor del 50 $\%$ de los adolescentes entre 15 y 19 años tenía vida sexual activa, el $25 \%$ de ellos se embarazaba y el $60 \%$ de las gestaciones ocurrían en los primeros seis meses posteriores al inicio de las relaciones sexuales. Además, entre el $60 \%$ y el $70 \%$ de esos embarazos no fueron deseados (2). En la actualidad, de acuerdo con la Encuesta Nacional de Demografía y Salud (ENDS) 2015, el 17,4 \% de las mujeres de 15 a 19 años ya es madre o está embarazada del primer hijo. El porcentaje para el inicio de la vida sexual antes de los 15 años fue del $31,1 \%$ en las mujeres de 20 a 24 años y de 16,3\% para los hombres de ese mismo rango de edad (3), condición que los hace vulnerables a contraer infecciones de trasmisión sexual (ITS), entre ellas el VIH/sida. Según la OMS, "se estima que, anualmente, unos 357 millones de personas contraen alguna de las cuatro infecciones de transmisión sexual (ITS) siguientes: clamidiasis, gonorrea, sífilis o tricomoniasis" (4).

La planificación familiar se ha desarrollado como pilar fundamental a nivel de la salud sexual y reproductiva. Se asocia con la reducción de los índices de mortalidad materno-perinatal, previniendo que las gestantes mueran durante el embarazo, parto o puerperio y proporcionándoles los elementos necesarios para evitar los riesgos que se presentan, además de brindarles la información requerida 
ISSN-PRINT

1794-9831

E-ISSN 2322-7028

Vol. 15 No. 1

Ene - Jun 2018

Cúcuta, Colombia que les permita aplazar la gestación hasta que se encuentren preparadas mental, física, emocional y económicamente (5).

En los últimos años, en América Latina y Asia se ha incrementado el uso de métodos de planificación familiar, de un $54 \%$ en 1990 a un $57.4 \%$ en 2014, pero aun así no deja de existir una problemática entre los jóvenes debido al mal uso que se les da a los anticonceptivos o por la poca frecuencia con que utilizan estos métodos en sus relaciones sexuales (6).

A medida que se acorta la edad de ingreso de la población estudiantil en las distintas instituciones universitarias de la ciudad, se visualiza con preocupación la falta de conocimientos acerca de la salud sexual y reproductiva y el mal uso de los métodos anticonceptivos, derivando esta situación en problemas subyacentes tales como embarazos, abortos e infecciones de transmisión sexual (7).

Frente a este panorama, la educación juega un papel muy importante en cuanto a las prácticas de salud sexual y reproductiva, puesto que muchas veces los jóvenes tienen conductas sexuales riesgosas por el desconocimiento o falta de información acerca de este tema, fenómeno que les impide protegerse ante un embarazo, ITS o las complicaciones que éstas conllevan, tales como los abortos, las muertes maternas, la deserción universitaria y las concepciones prematrimoniales, entre otras.

Según un estudio realizado en la Escuela Superior Politécnica de Chimborazo, Ecuador, durante los años 2009-2010 (8), que indagó acerca de los conocimientos, prácticas y comportamientos de los estudiantes en cuanto a la sexualidad, se identificó un amplio déficit de conocimientos, referenciando para ello nunca haber utilizado un preservativo durante sus relaciones sexuales y, en su lugar, utilizar métodos caseros no eficaces, además de tomar en cuenta las diferentes creencias y costumbres que tienen sobre el tema.

En Colombia, un estudio realizado en Cartagena (9) con 979 estudiantes reportó que el $8.5 \%$ de los encuestados presentaba embarazo adolescente -el $80 \%$ no deseado y el $7 \%$ como producto de una relación sexual bajo efectos del alcohol-. Por otro lado, se encontró que la prevalencia del aborto fue del $6.7 \%$, lo cual indica que aún continúa existiendo falta de conocimiento de los estudiantes sobre su salud sexual y reproductiva.
En la Universidad del Cauca se realizó un estudio en el año 2011 (10), el cual reporto que el $89.3 \%$ de los estudiantes eran sexualmente activos -en mayor proporción los hombres-. En cuanto a los métodos anticonceptivos, el más usado fue el condón, con el $82.9 \%$, aunque se presentaron algunas falencias en su uso. La píldora anticonceptiva de emergencia fue usada por el $90.5 \%$ de los estudiantes encuestados, aunque con desconocimiento de sus efectos.

De acuerdo con lo anterior, se evidenció que la salud sexual y reproductiva de los adolescentes universitarios es un tema que necesita de gran trabajo y atención primaria por parte de los entes gubernamentales de salud, puesto que la problemática no se presenta solamente en Colombia, sino que acontece en diferentes países y no precisamente se centra en una temática específica sino que abarca diferentes aspectos.

Actualmente, con el cambio de las telecomunicaciones y una mayor permanencia de los jóvenes en las redes sociales e internet, sin duda alguna se pone de manifiesto que el conocimiento no es universal y no se interpreta de la misma manera. De acuerdo con González (11) y Valencia (12), estamos en la época en que se debería mejorar la calidad y cobertura de los servicios de salud reproductiva mediante procesos de intervención, educación y capacitación que podrían mejorar el proyecto de vida de los jóvenes y adolescentes del territorio colombiano.

Algunas universidades, como la que se aborda en este estudio, ya han implementado espacios en donde se fomenta el desarrollo de programas de promoción de la salud y prevención de la enfermedad, en los cuales se incluyen los servicios especializados en salud sexual y reproductiva y se busca disminuir el problema existente, pero, lamentablemente, a este servicio acuden pocos estudiantes debido al desconocimiento, a factores sociales y a estereotipos.

Este estudio cuantitativo descriptivo busca describir el conocimiento y las prácticas sobre la salud sexual y reproductiva en los estudiantes de pregrado de una institución universitaria del suroccidente colombiano, de tal manera que se puedan reconocer y analizar los factores que ponen en riesgo a la población estudiantil $\mathrm{y}$, desde esa perspectiva, fortalecer el enfoque y la atención de enfermería en los servicios universitarios 
de salud sexual y reproductiva, teniendo en cuenta que esta profesión realiza el análisis y la atención de los jóvenes en el contexto de su salud sexual, como componente fundamental del bienestar de un individuo, garantizando la formulación de los denominados derechos sexuales y reproductivos que buscan el máximo nivel de salud posible en todos los aspectos relacionados con la sexualidad y la reproducción, además de garantizar de forma fundamental el derecho a la vida (13).

\section{Objetivos}

\section{Objetivo general}

Describir el conocimiento y las prácticas sobre salud sexual y reproductiva en los estudiantes de pregrado de I y II semestre de una universidad del suroccidente colombiano

\section{Objetivos específicos}

- Identificar los conocimientos sobre la salud sexual y reproductiva en los estudiantes de pregrado de I y II semestre

- Identificar las prácticas de autocuidado que realizan los estudiantes de pregrado I y II semestre en relación con la salud sexual y reproductiva

- Determinar la frecuencia del uso de métodos anticonceptivos en los estudiantes.

- Identificar el grado de relación o asociación entre los conocimientos y las prácticas en salud sexual y reproductiva de los estudiantes.

\section{Materiales y Métodos}

Se realizó un estudio cuantitativo, descriptivo y prospectivo de corte transversal. Para el cálculo de la muestra se tuvo en cuenta una prevalencia de uso de métodos de planificación familiar de 48,2\% (14), un error del $5 \%$ y un nivel de confianza del $95 \%$, para un tamaño de muestra de 346 estudiantes, más un 20 $\%$ adicional de no respuesta, calculando así un total final de la muestra de 415 individuos a estudio.

Para determinar la forma de diligenciamiento de la encuesta por parte de los estudiantes, se realizó un muestreo de tipo probabilístico aleatorio estratificado por afijación proporcional para cada Facultad de la
Universidad, de acuerdo con el número de estudiantes para cada programa. El proceso de reemplazo en caso de que el estudiante elegido no estuviera presente o decidiera no participar en el estudio, contempló escoger al estudiante siguiente en la lista. Fueron utilizadas encuestas autodirigidas de forma anónima, con previo consentimiento informado.

Se elaboró el instrumento tipo encuesta estructurada de selección, teniendo como referente el formato de consejería del programa de salud sexual y reproductiva de la División de Salud Integral y Desarrollo Humano perteneciente a la Vicerrectoría de Cultura y Bienestar de la Universidad (15). También se tuvieron en cuenta los objetivos del presente trabajo y la encuesta de la Organización Panamericana de la Salud denominada Protocolos de Investigación para el Estudio de la Salud Sexual y Reproductiva de los Adolescentes Varones y Hombres Jóvenes en América Latina $(16,17)$.

Para determinar los grupos de edad se tuvo en cuenta la Norma Técnica para la Detección Temprana de las alteraciones del desarrollo del joven de 10 a 29 años (18), de la siguiente manera: Adolescencia temprana o inicial (10-13 años), Adolescencia media (14-16 años), Adolescencia final o tardía (17-21 años), Adulto joven (21-24 años) y Adulto tardío (25-45 años).

La encuesta para la recolección de la información constó de 3 módulos: el primero con 12 ítems y preguntas relacionadas con datos sociodemográficos, gineco-obstétricos, conocimientos acerca de los derechos sexuales y reproductivos, anatomía del aparato reproductor femenino y masculino, factores para adquirir ITS o cáncer del cuello uterino o cáncer de mama, consecuencias del aborto y embarazo a temprana edad, política nacional de salud sexual y reproductiva, además de la ruta de atención al abuso sexual, los cuales se tabularon de acuerdo con la información brindada por los participantes, en una escala tipo Likert, describiendo el nivel de conocimientos como bueno, regular o malo.

Para explorar las prácticas, un segundo módulo incluyó 24 preguntas semicerradas que se tabularon de acuerdo con la persistencia de las respuestas brindadas, relacionadas con el autocuidado de la salud sexual y reproductiva de los estudiantes. Aquí se abordaron preguntas sobre violencia de género en las cuales el participante discriminaba si había sido 
ISSN-PRINT

1794-9831

E-ISSN 2322-7028

Vol. 15 No. 1

Ene - Jun 2018

Cúcuta, Colombia víctima de algún tipo de violencia y si ésta había sido denunciada.

La encuesta se sometió a revisión por parte de expertos en el área de salud sexual y reproductiva (Enfermeros expertos docentes con grado de maestría de la Universidad objeto de estudio) y posteriormente se realizaron ajustes al instrumento de acuerdo con la prueba piloto que se realizó con el $10 \%$ de la muestra en la población de universitarios de I-II semestre que no se tuvo en cuenta para la realización de la investigación.

Las variables cualitativas se analizaron e interpretaron con medidas de proporciones, enunciadas en términos de frecuencia y porcentajes. Las variables cuantitativas se analizaron con medidas de tendencia central y de dispersión. Para la tabulación y análisis de los datos se utilizó el paquete estadístico Excel versión 2010 y SPSS Versión 22. Para determinar el significado de la asociación de las variables se utilizó la prueba de Chi cuadrado, con una confiabilidad del $95 \%$.

Como se trató de una investigación con estudiantes universitarios, en los que podría existir subordinación, el proyecto se presentó al Comité de Ética en Investigación de la Universidad, siendo avalado con el código 4564. Se realizó una sesión explicativa del estudio, luego de la cual cada estudiante firmó el consentimiento informado y diligenció la encuesta.

\section{Resultados}

La recolección de la información se realizó durante los meses de febrero y marzo del año 2016. Las limitaciones se relacionaron con la obtención de la muestra -teniendo en cuenta que la muestra inicial fue de 415 estudiantes- debido a la dificultad de recolección de datos en algunos programas como Ingeniería Forestal de la Facultad de Ciencias Agrarias, Ciencias Políticas de la Facultad de Derecho, Artes Plásticas de la Facultad de Artes y Lenguas Modernas, pertenecientes a la Facultad de Humanidades, en virtud de los diferentes horarios de clase y laboratorios en estas facultades, obteniendo 363 estudiantes como muestra final.

\section{Caracterización sociodemográfica}

En el primer periodo académico del 2016 se aplicaron 363 encuestas, distribuyéndose de manera proporcional en cada facultad y carreras de la Universidad. Respecto a la población estudiada, se encontró un predominio masculino, el 94,2\% de los estudiantes son solteros, el $60.9 \%$ de ellos profesa la religión católica, el 26,2\% no pertenece a ninguna religión y el $12,9 \%$ profesa el cristianismo. Además, el $78 \%$ procede de la zona urbana de diferentes Departamentos de Colombia, siendo el de mayor prevalencia el Departamento del Cauca con un 79,1 $\%$, seguido del Departamento de Nariño con un 9,6 $\%$ (Ver Tabla 1). 
Ivett Adriana Herrera Zuleta,Jonathan Fernando Reyes Salazar, Katherine Patricia Rojas Velasco, Magda Lizeth Tipán, Carlos Andrés Torres López, Lina Marcela Vallejo Trujillo, Luis Felipe Zemanate Ordoñez.

Tabla 1. Distribución porcentual sobre datos sociodemográficos en estudiantes de I y II semestre de pregrado de la Universidad del Cauca, 2016.

\begin{tabular}{|c|c|c|c|}
\hline \multicolumn{2}{|c|}{ DATOS SOCIODEMOGRAFICOS DE POBLACION ESTUDIDA } & \multirow{2}{*}{$\begin{array}{c}\text { NUMERO } \\
184\end{array}$} & \multirow{2}{*}{$\frac{\%}{50,7 \%}$} \\
\hline \multirow{3}{*}{\multicolumn{4}{|c|}{$\begin{array}{l}\text { FEMENINO } \\
\qquad \text { PROMEDIO: } 19,1 \text { AÑOS } \pm 3 \text { AÑOS }\end{array}$}} \\
\hline & & & \\
\hline & & & \\
\hline ADOLESCENCIA MEDIA & 14-16 años & 22 & $6 \%$ \\
\hline ADOLESCENCIA TARDIA & 17-21 años & 295 & $81 \%$ \\
\hline ADULTO JOVEN & 22-24 años & 30 & $8 \%$ \\
\hline ADULTO TARDIO & $25-45$ años & 16 & $4 \%$ \\
\hline \multirow[t]{3}{*}{ ESTADO CIVIL } & SOLTERO & 342 & $94,2 \%$ \\
\hline & CASADO & 5 & $1,4 \%$ \\
\hline & UNION LIBRE & 16 & $4,4 \%$ \\
\hline \multirow[t]{3}{*}{ ESTRATO } & $1 \mathrm{Y} 2$ & 214 & 59,0 \\
\hline & $3 \mathrm{Y} 4$ & 137 & $37,7 \%$ \\
\hline & 5 Y 6 & 12 & $3,3 \%$ \\
\hline \multirow[t]{4}{*}{ RELIGION } & CATOLICO & 221 & $60,9 \%$ \\
\hline & CRISTIANO & 42 & $11,6 \%$ \\
\hline & NINGUNA & 95 & $26,2 \%$ \\
\hline & OTRO & 5 & $1,4 \%$ \\
\hline \multirow[t]{2}{*}{ PROCEDENCIA } & RURAL & 78 & $21,5 \%$ \\
\hline & URBANO & 285 & $78,5 \%$ \\
\hline \multirow[t]{11}{*}{ DEPARTAMENTO } & CAUCA & 287 & $79,1 \%$ \\
\hline & NARIÑO & 35 & $9,6 \%$ \\
\hline & VALLE DEL CAUCA & 5 & $1,4 \%$ \\
\hline & HUILA & 18 & $5,0 \%$ \\
\hline & ARAUCA & 1 & $0,3 \%$ \\
\hline & CASANARE & 1 & $0,3 \%$ \\
\hline & CAQUETA & 2 & $0,6 \%$ \\
\hline & PUTUMAYO & 11 & $3,0 \%$ \\
\hline & ANTIOUIA & 1 & $0,3 \%$ \\
\hline & TOLIMA & 1 & $0,3 \%$ \\
\hline & TOTAL & 363 & $100 \%$ \\
\hline
\end{tabular}

Fuente: Base de datos SSYR EUP2016

\section{El conocimiento sobre salud sexual y reproductiva}

Acerca de los conocimientos sobre salud sexual y reproductiva, se encontró que el $62 \%$ de los estudiantes tiene un nivel de conocimiento regular, el $14 \%$ tiene un nivel bueno y el $24 \%$ tiene conocimiento deficiente. Según temas específicos sobre salud sexual y reproductiva, el $86 \%$ de los estudiantes afirmó conocer las consecuencias de un embarazo a temprana edad, seguido de la función del aparato reproductor femenino y masculino $(82 \%$,), la transmisión de las ITS y VIH/SIDA $(75,5 \%)$, la ruta de atención al abuso sexual $(64,5 \%)$ seguido de la Política de Salud Sexual Reproductiva del país $(54,8 \%)$ y los derechos de salud sexual y reproductiva (45\%.) (Ver Grafica 1.) 
ISSN-PRINT

1794-9831

E-ISSN 2322-7028

Vol. 15 No. 1

Ene - Jun 2018

Cúcuta, Colombia
Prácticas de los universitarios en relación con la salud sexual y reproductiva

Respecto al inicio de actividad sexual y uso de métodos de planificación familiar (MPF), el $78 \%$ de la población manifestó que había iniciado su vida sexual -con mayor prevalencia el género masculino $(58,1 \%)$ a una edad media de 16 años, en ambos sexos. El $71 \%$ refirió haber utilizado algún método de planificación familiar en su primer encuentro sexual, principalmente el condón (70 \%), cuya iniciativa de uso fue por decisión de ambos $(72 \%)$ y por decisión propia (22 $\%)$. Dentro de la población que no utilizó ningún MPF en su primera relación sexual (29 \%), las razones más frecuentes fueron no considerar necesario o importante utilizar estos métodos (59\%), desconocer los MPF (18 $\%)$ y otras razones como no tener dinero y/o no tener planeado una relación sexual ese día (11\%).

El $96 \%$ de los estudiantes tuvo entre 1 y 3 compañeros sexuales en el último año, mientras que el restante $4 \%$ tuvo más de 3 compañeros sexuales. Las principales razones por las cuales los estudiantes deciden tener relaciones sexuales fueron: placer (46\%), amor a la pareja $(27 \%)$ y un $11 \%$ lo hace por una razón social, ya sea porque se dio la oportunidad, porque estuvo bajo efectos de sustancias psicoactivas o porque la mayoría de los compañeros lo realizan.

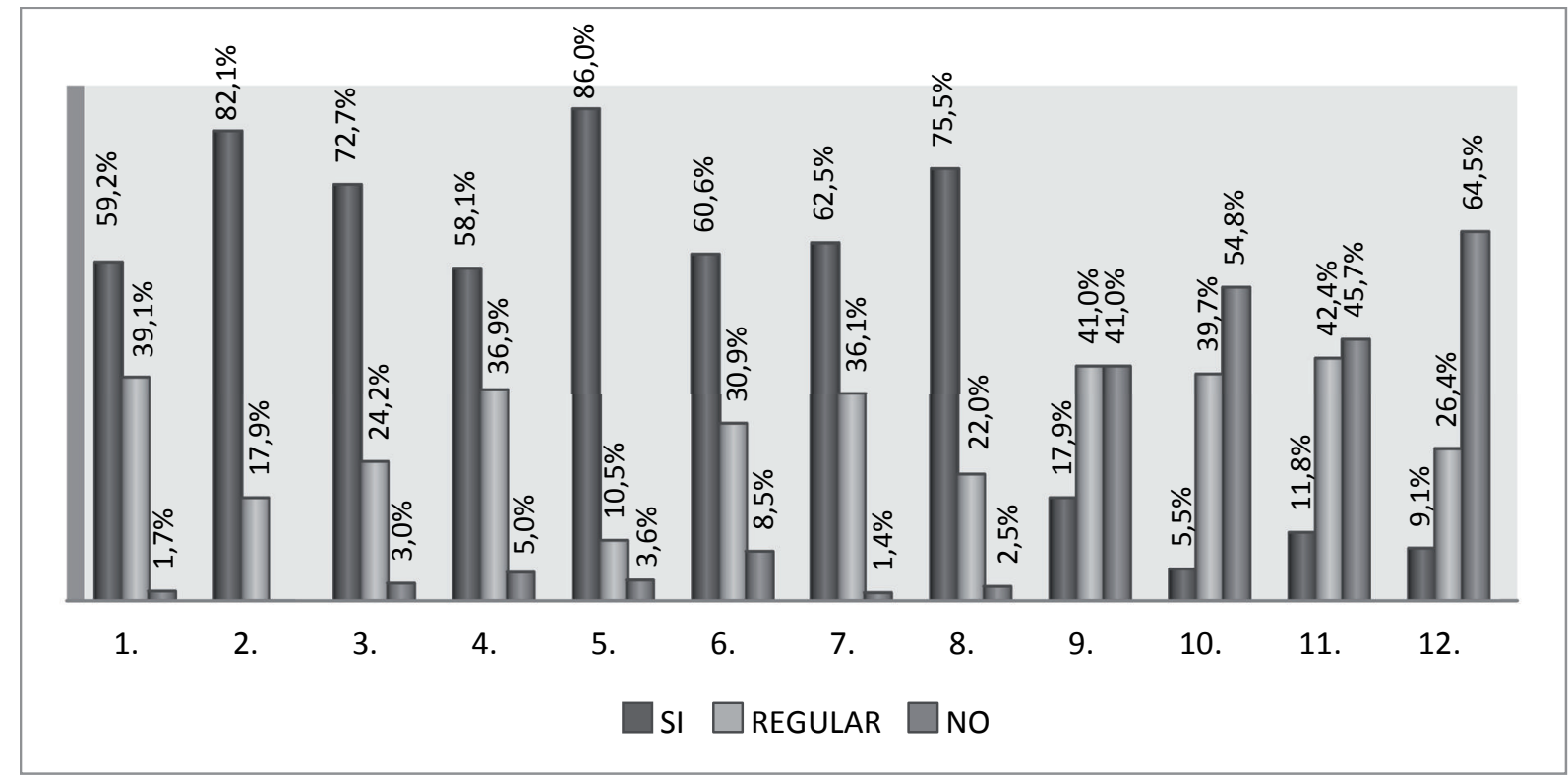

1. Anatomía del aparato reproductor femenino y masculino 2. Función del aparato reproductor masculino y femenino 3. Métodos de planificación familiar 4. Cómo se utilizan los métodos de planificación familiar 5. Consecuencias de un embarazo a temprana edad 6. Consecuencias del aborto 7. Cuáles son las ETS y VIH/SIDA 8.Transmisión de ETS y VIH/SIDA 9.Factores de riesgo para contraer cáncer de cérvix 10. Política de SSYR del país 11. Los derechos de SSYR 12.La ruta de atención al abuso sexual.

Grafica 1. Distribución porcentual sobre conocimientos en salud sexual y reproductiva en estudiantes de primer y segundo semestre de pregrado de la Universidad del Cauca, 2016.

Fuente: Base de datos Salud Sexual y Reproductiva en estudiantes de I y II semestre de la Universidad del Cauca, 2016.

Frecuencia y uso de los métodos de planificación familiar.Se encontró que el $76 \%$ de los estudiantes utilizan un método de planificación familiar (MPF), de los cuales el principal se clasifica como método de barrera con el $62 \%$.

El $23 \%$ utilizó dos MPF, siendo predominante el uso de anticonceptivo de barrera en combinación con el hormonal (18\%). En cuanto al anticonceptivo de emergencia, el 12,3\% de los estudiantes refirió utilizar la píldora de emergencia.

En cuanto a la frecuencia de uso de los MPF en sus relaciones sexuales, el $56 \%$ los utiliza siempre, el $22,3 \%$ casi siempre, el 5,3\% casi nunca y el $3 \%$ manifiesta nunca utilizarlos. E1 $51 \%$ de los estudiantes siempre utiliza el condón en sus relaciones sexuales $(p=0,000)$. 


\section{El embarazo en los universitarios}

El $11 \%$ de los estudiantes que había iniciado relaciones sexuales en algún momento de su vida tuvo embarazo. El $30 \%$ de estos embarazos finalizaron en aborto y el $70 \%$ lo continuaron -de este último porcentaje, el $56 \%$ fueron embarazos no deseados y el $44 \%$ deseados-. Para el caso de la interrupción del embarazo, el 62,5 \% terminó en aborto inducido, el $70 \%$ producido por medicamentos y el 37,5 \% correspondió a aborto espontaneo.

\section{Prácticas de autocuidado}

Con relación a las prácticas de autocuidado en la población femenina, el $19 \%$ se realiza el autoexamen de mama, teniendo una frecuencia de realización mensual el $48,6 \%$ y semestral el $20 \%$, mientras que el 7\% refirió no realizarla regularmente. Respecto a la vacunación contra el virus del papiloma humano, el $44 \%$ de la población afirmó haberse aplicado esta vacuna, en tanto que el $56 \%$ manifestó no haberlo realizado. Adicionalmente, se encontró que el 52,5\% de las mujeres no se había realizado la citología.

En la población masculina, el 6,3\% se realizó el autoexamen de testículo, con una frecuencia mensual del $30,4 \%$, semanal del $26 \%$ y el $17,4 \%$ refiere no realizarlo con mucha frecuencia.

\section{Violencia de género}

El 7,4 \% de los estudiantes manifestó haber sido víctima de violencia, siendo más prevalente en mujeres $(77.8 \%$; $=0,002)$. Los tipos de violencia que se desarrollaron fueron los relacionadas con maltrato psicológico (37\%), abuso sexual (26\%) y maltrato físico (22\%). El 77,8 \% de los estudiantes que experimentó algún tipo de violencia no lo denunció ante las autoridades judiciales, refiriendo como razón principal el miedo a represalias $(61,9 \%)$ y porque no lo consideró de gran importancia (19\%).

\section{Análisis bivariado}

Se realizaron cruces de variables, para lo cual se utilizó la prueba de significancia Chi cuadrado.

Con relación al nivel de conocimiento y a los datos sociodemográficos, se encontró que el nivel de conocimientos sobre SSYR está asociado al género, puesto que el género femenino reportó tener mejor conocimiento que el masculino $(\mathrm{p}=0,001)$, mientras que no se encontró asociación entre el nivel de conocimiento con: el grupo de edad $(\mathrm{p}=0,0860)$, el nivel socioeconómico $(\mathrm{p}=0,072)$, la procedencia $(\mathrm{p}=0,514)$ o la religión $(\mathrm{p}=0,316)$.

Para el inicio de vida sexual versus género, se encontró asociación significativa con predominio en el género masculino $(57,6 \% ; \mathrm{p}=0,000)$, es decir, que para este estudio ser del género masculino aumenta las posibilidades de iniciar vida sexual de forma temprana. Para la asociación entre el grupo de edad vs compañeros sexuales en el último año, se destaca que la mayoría de los estudiantes que tiene más de una pareja sexual se encuentra en la etapa de adolescencia tardía ( $\mathrm{p}=0,049)$, por lo cual se infiere que estar en una edad entre 17 a 21 años aumenta la posibilidad de tener más de una pareja sexual, convirtiéndose en un factor de riesgo para contraer ITS. Con relación al grupo de edad vs antecedente de embarazo, se reportó que el 47 $\%$ de los embarazos se presentaron en la adolescencia tardía, con asociación significante $(\mathrm{p}=0,003)$.

Con relación al género, se encontró asociación estadísticamente significativa en uso de píldora de emergencia con predominio en género masculino $(65,7 \% ; p=0,002)$, víctima de violencia con mayor frecuencia de violencia en las mujeres $(77,8 \%$; $\mathrm{p}=0,002)$ y autoexamen de mama con predominio en mujeres del 95,7\% $(\mathrm{p}=0,000)$.

\section{Discusión}

De acuerdo con los resultados obtenidos, se pudo evidenciar que los conocimientos acerca de salud sexual y reproductiva son, en su mayoría, regulares (62 \%). Las áreas o temáticas en donde se encontró mayor conocimiento fueron: las consecuencias de un embarazo a temprana edad, la función de aparato reproductor y la transmisión de ITS y VIH/SIDA. Por el contrario, hay un desconocimiento general acerca de derechos sexuales y reproductivos y política de salud sexual y reproductiva del país. Esta situación es similar a la que se encontró en el estudio de Mantilla et al. (19), donde se demostró que, para los adolescentes, los derechos sexuales y reproductivos no eran considerados importantes, ni tampoco los conocimientos o las prácticas al respecto. Esta situación es preocupante debido a que, si no se tiene un conocimiento suficiente sobre este tema, los estudiantes están más propensos a la vulneración de sus derechos y, además, no harán uso de las políticas 
ISSN-PRINT

1794-9831

E-ISSN 2322-7028

Vol. 15 No. 1

Ene - Jun 2018

Cúcuta, Colombia estatales y universitarias puestas a su disposición para el goce de una sexualidad responsable (20).

Respecto a las prácticas, el inicio temprano de relaciones sexuales es un factor muy preocupante debido a que se encontró que el $77 \%$ de los estudiantes ya había iniciado relaciones sexuales, con un promedio de inicio de la actividad sexual de 16 años $(+o-2)$. Esta situación es comparable con lo demostrado por Acosta et al. (21), en el año 2010, donde se observó que el 80 \% de la población estudiantil informó haber iniciado relaciones sexuales con un promedio de inicio de 14 años.

Es de resaltar que, a pesar de la corta edad de inicio de actividades sexuales, en este estudio un alto porcentaje (71 \%) utilizó algún método de planificación, cuya iniciativa de uso fue por decisión de ambos. Respecto al método usado, predomino el condón, situación muy favorable ya que el uso de este dispositivo no sólo evita los embarazos sino que, además, protege contra las ITS. El porcentaje restante de población tendrá un factor de riesgo, ya que el inicio temprano de relaciones sexuales sin una adecuada protección trae consigo una serie de riesgos inherentes, tales como embarazos no deseados y enfermedades de trasmisión sexual, factores que, sin duda, afectan negativamente el desarrollo de la personalidad y el proyecto de vida de los estudiantes.

De otra parte, a pesar del inicio temprano de relaciones sexuales, los embarazos no son una problemática en la población encuestada, puesto que se registró apenas un $11 \%$ de embarazos, situación similar a lo encontrado por Gómez et al. (14), en 2014, en cuyo estudio el embarazo se presentó en un 12,3\%. Aquí lo realmente preocupante radica en la terminación de estos embarazos, ya que en este estudio se evidenció que el $41 \%$ terminó en aborto -el $75 \%$ de esos abortos fue inducido por medicamentos, convirtiéndose esto en una problemática si se toma en cuenta el fácil acceso de los estudiantes a ellos-, sumado a que estos procedimientos se hacen en condiciones no adecuadas y, generalmente, sin el acompañamiento de personal de salud idóneo, lo cual puede acarrear graves consecuencias para la salud y la integridad de la mujer, tales como histerectomía o incluso la muerte. Por lo tanto, es necesario dar educación a los estudiantes acerca de las consecuencias del aborto.

La disponibilidad de métodos anticonceptivos altamente eficaces ha ofrecido a hombres y mujeres la posibilidad de planificar su vida familiar, permitiendo un mejor desarrollo personal y una vida sexual más plena. Sin embargo, no todas las personas tienen el mismo nivel de conocimiento ni de acceso a ellos (22). Al indagar en los estudiantes sobre la frecuencia con que usan métodos anticonceptivos en sus relaciones sexuales, el $50.58 \%$ aseguró usarlo en todas sus relaciones sexuales, mientras que el 2,9 \% afirmó nunca utilizarlos debido a que no lo consideran importante. Esta frecuencia de uso es alta si se compara con el estudio de Camacho y Pabón (23), en 2014, en el cual se encontró que apenas un 25 \% utilizó algún método anticonceptivo. Este estudio consideró que la alta frecuencia de uso de métodos de barrera $(61,65 \%)$, se ha convertido en un factor protector para los estudiantes que se puede ver reflejado en el bajo porcentaje de embarazos (11\%) y la baja incidencia de infecciones de transmisión sexual ITS (1\%).

Los embarazos no deseados han sido siempre una problemática nacional, debido a las consecuencias que traen consigo. En primera medida, se encuentra el aborto, como lo relata Ana Langer en su estudio de impacto sobre la salud y la sociedad en América Latina y el Caribe (24).

El porcentaje de embarazos no deseados a nivel nacional es de 50,5\% según la Encuesta Nacional de Demografía y Salud, ENDS 2015, el cual ha venido disminuyendo en los últimos años en virtud de que hay un mayor acceso a los métodos anticonceptivos (25). Aunque en los sujetos de este estudio el porcentaje de embarazos es bajo, con apenas $11 \%$, es importante señalar que el $60 \%$ de éstos fueron no deseados, lo cual supera la cifra nacional.

Analizando las estadísticas nacionales de embarazos no deseados, puede plantearse que la disminución de éstos está relacionada con el incremento y buen uso de métodos anticonceptivos, práctica que debe fomentarse en la consulta de enfermería, durante la consejería en salud sexual y reproductiva. Así como, acerca del control de los métodos anticonceptivos, el estudio mostró que son muy aceptados por los universitarios, principalmente por su bajo costo y alta efectividad.

Debido a que la violencia es un tema fundamental en el entorno de la salud sexual y reproductiva, también se analizó en este estudio, encontrándose presente en el 8 $\%$ de los encuestados, resultado similar al de Rangel et al, en el año 2012, en donde el $12 \%$ ha sufrido en un momento de su vida algún tipo de violencia 
(26). Aunque este es un valor bajo, no deja de ser preocupante e intolerable la presencia de violencia en los universitarios, convirtiéndose en un tema que debería ser objeto de un estudio más detallado que permita identificar los factores que intervienen en dicha violencia y que permitan tomar acciones tendientes a la disminución de este fenómeno.

El factor género juega un papel importante en cuanto a violencia se refiere y este estudio no fue la excepción. Se determinó que la mayor frecuencia de violencia se dio en el género femenino, con resultados equivalentes a los datos obtenidos en los estudios de Mohamed, (27), en el año 2014, en los cuales obtuvieron puntuaciones más altas las mujeres que los hombres. Caso similar acontece en el estudio de Rangel, en el año 2012, que muestra una prevalencia del $68 \%$ de violencia contra la mujer (28).

Otra situación igualmente preocupante es la falta de denuncia por parte de los encuestados que fueron víctimas de violencia. Apenas el $19 \%$ denunció, siendo el miedo y el desconocimiento de los derechos y las rutas de atención a la violencia, las principales causas de no denuncia. Por lo tanto, se hace necesario informar a los estudiantes, desde las aulas de clase, sobre sus derechos, fomentando en ellos la cultura de la no tolerancia con la violencia.

En cuanto al tipo de violencia, sobresale principalmente el psicológico, seguido de la violencia de tipo sexual, hallazgos semejantes a los obtenidos en el estudio de Gómez (14), del año 2014, donde se registró mayor prevalencia en la violencia de tipo físico $(21,6 \%)$ seguida de la de tipo sexual. Lo más relevante y concluyente es que estas conductas no son denunciadas y continúan siendo una epidemia en la sociedad (29).

Con relación a las prácticas de autocuidado tanto en mujeres como en hombres, el $19 \%$ de se realiza el autoexamen de mama, mientras que el $13 \%$ de los hombres se practica el examen de testículo. Estos resultados son diferentes a los reportados por Cerezo et al. en el año 2011, en donde el $55 \%$ de las mujeres se había realizado el autoexamen de mama y el $32 \%$ de los hombres refirió haberse practicado el autoexamen de testículo (28). Esta situación pone de manifiesto los factores de riesgo a los cuales se encuentra expuesta esta población, debido a que estas prácticas de autocuidado son pruebas de tamizaje que ayudarían a un diagnóstico temprano para un posible cáncer.
En relación con las prácticas sexuales de riesgo, en Colombia se ha encontrado que los hombres inician su vida sexual más temprano que las mujeres $(30,31)$, situación similar a la encontrada en este estudio, donde los hombres iniciaron su vida sexual a menor edad que las mujeres. Esta conducta se debe principalmente, como lo afirman Rodríguez y Álvarez, a que el varón asume una posición más irracional y la mujer se deja influenciar más por los prejuicios sociales que la discriminan (30). Por lo tanto, es necesario actuar sobre estos factores culturales que influyen negativamente en el comportamiento sexual de los universitarios, exponiéndolos a todos los factores de riesgo que implica el inicio precoz de las relaciones sexuales sin los conocimientos apropiados que garanticen el ejercicio responsable de la sexualidad.

\section{Conclusiones}

- Enfermería, como líder en la promoción de la salud y la prevención de la enfermedad, debe continuar con el mejoramiento del programa de salud sexual y reproductiva brindado en las instituciones de atención a jóvenes y adolescentes, específicamente en bienestar universitario, en donde se debe fortalecer el programa educativo, enfatizando en la consejería y servicios de ayuda en situaciones de vulnerabilidad.

- Factores como el género, el estado civil, la adolescencia tardía y la religión llevan a los estudiantes a iniciar sus relaciones sexuales de forma temprana. No obstante, estos datos sociodemográficos no fueron significativos para este estudio.

- Los estudiantes participantes en la investigación tienen poco conocimiento sobre la salud sexual y reproductiva, aunque reconocen las consecuencias de un embarazo a temprana edad y de las infecciones de transmisión sexual.

- Los estudiantes universitarios utilizan, al menos, un método de planificación familiar e inician su vida sexual con edades alrededor de los 15 años para los hombres y los 16 años para las mujeres, teniendo algunos de ellos múltiples parejas -aproximadamente entre 1 y 3 compañeros (as) sexuales al año-, lo cual los convierte es una población susceptible de adquirir ITS, VIH/ SIDA y embarazos no deseados que podrían terminar en un aborto inducido
E-ISSN 2322-7028

Vol. 15 No. 1

Ene - Jun 2018

Cúcuta, Colombia 
ISSN-PRINT

1794-9831

E-ISSN 2322-7028

Vol. 15 No. 1

Ene - Jun 2018

Cúcuta, Colombia
- Se evidenció que una pequeña población de estudiantes universitarios, en su mayoría mujeres, en algún momento de su vida ha sufrido algún tipo de violencia, principalmente psicológica, fenómeno que no es denunciado por miedo a represalias.

\section{Conflicto de intereses}

Los autores declaran no tener ningún conflicto de intereses.

\section{Referencias Bibliográficas}

1. Gómez Camargo DE, Ochoa Díaz MM, Canchila Barrios CA, Ramos Clason EC, Salguedo Madrid GI, Malambo García DI.. Salud sexual y reproductiva en estudiantes universitarios de una institución de educación superior en Colombia. Rev. salud pública [Internet]. 2014 [consultado 03 Mar 2017]; 16(5):660-672. Disponible en: http://www.scielo.org.co/pdf/rsap/v16n5/v16n5a02.pdf

2. González Pérez L, González Lucas N, Mena Fernández M, Navarro Padrón A, Martín Linares X. Salud Sexual y Reproductiva en Estudiantes Universitarios. ¿Modo y Estilo de Vida Saludables?, Un diagnóstico. Rev Hum Med [Internet] 2003, [Consultado 03 Marzo 2017]; 3(1). Disponible en: http:// scielo.sld.cu/scielo.php?script $=$ sci_arttext\&pid $=\mathrm{S} 1727-81202003000100001 \& \operatorname{lng}=\mathrm{es}$.

3. Ministerio de Salud y Protección Social. Boletín de Prensa No 261 de 2016 Disponible en: https://www. minsalud.gov.co/Paginas/Ministerio-de-Salud-y-Profamilia-entregan-resultados-de-la-ENDS-2015. aspx

4. OMS. Infecciones de trasmisión sexual. 2016. Disponible en: http://www.who.int/mediacentre/ factsheets/fs110/es/

5. Ministerio de Salud. Planificación familiar, derecho de todas y todos. Perú. [Internet] 2012. [Consultado 09 Mar 2017] Disponible en: http://www.minsa.gob.pe/portada/Especiales/2012/planfam/ introduccion.asp

6. OMS. Planificación familiar. Centro de Prensa. 2015. Nota descriptiva $\mathrm{N}^{\mathrm{o}} 351$. Disponible en: http:// www.who.int/mediacentre/factsheets/fs351/es/

7. Trejo O, Perla M, Moreno C, Nacias A, Valdez E. y Col. Conocimiento y comportamiento sexual en adolescentes. área académica. Ciencias de la Salud Universidad Autónoma de Zacatecas. Rev, Cuba Enferm 2011; 27(4): 273-280.

8. Aeteros Hernández RC, Pérez Pinero J, Sanabria Ramos G. Vivencias de la sexualidad en estudiantes universitarios. Rev Cubana Salud Pública. 2013; 39(1):915-928.

9. Díaz Cárdenas S, Arrieta Vergara K, González Martínez F. Prevalencia de actividad sexual y resultados no deseados en salud sexual y reproductiva en estudiantes universitarios en Cartagena, Colombia Revista Colombiana de Obstetricia y Ginecología. 2014; 65(1):22-31

10. Chávez Gómez MS. Conocimientos, Prácticas y uso de métodos anticonceptivos de estudiantes de la Universidad del Cauca. Popayán 2011 T.FCS- ENF 0335

11. González Román P. Antecedentes teóricos y empíricos del uso de métodos de planificación familiar. Rev.fac.cienc.econ. 2009; 17(2):171-82.

12. Valencia CP, Canaval GE. Factors predisposing, facilitating and strengthening condom use amongst university students in Cali, Colombia. Rev Salud Pública. 2012; 14(5):810-21

13. Osorio Castaño JH. La salud sexual y reproductiva como área para desarrollar la enfermería de práctica avanzada. Rev. cienc. cuidad. 2017; 14(2): 131-144

14. Gómez DE, Ochoa M, Canchila C, Ramos E, Salguedo G. y Malambo DI. Salud sexual y reproductiva en estudiantes universitarios de una institución de educación superior en Colombia. Rev. salud pública. [Internet] 2014. [Consultado 03 Marzo 2017]; 16(5):660-672. Disponible en: http://www.scielosp.org/ pdf/rsap/v16n5/v16n5a02.pdf.

15. Chavez M. Protocolo de Atención en consejería en salud sexual y reproductiva en adolescentes. División de Gestión de Salud Integral y Desarrollo Humano. Programa en Salud Sexual y Reproductiva. 
Universidad del Cauca. Popayán 2006.

16. Chávez M. Bastidas B. Orozco C. Merchán A. Conocimientos y prácticas de métodos anticonceptivos en una población universitaria en el año 2010. un estudio descriptivo. Rev. Facultad Ciencias de la Salud. [Internet] 2014. [Consultado 03 de Marzo de 2017]; 16(2). Disponible en: http://facultadsalud. unicauca.edu.co/revista/ojs2/index.php/rfcs/article/view/27/26

17. Lundgren R. Protocolos de investigación para el estudio de la Salud Sexual y Reproductiva de los adolescentes varones y hombres jóvenes en América Latina. Organización Panamericana de la Salud; 2010. [Internet]. Disponible en: http://www2.paho.org/hq/ dmdocuments/2010/ Protocolosinvestigacion-salud-sexual-y-reprod-adolescentesvarones-lac.pdf. Consultado julio de 2016.

18. República de Colombia, Ministerio de Salud, Dirección General de Promoción y Prevención. Norma Técnica para la Detección Temprana de las alteraciones del desarrollo del joven de 10 a 29 años. Disponible en: https://www.minsalud.gov.co/sites/rid/Lists/BibliotecaDigital/ RIDE/VS/ PP/7Deteccion\%20temprana\%20alteraciones\%20joven.pdf

19. Mantilla Uribe BP, Hakspiel Plata MC, Rincón Méndez AY, Smith Hernández D, Hernández Quirama A. Promoción de derechos sexuales y reproductivos en adolescentes de Bucaramanga, Floridablanca y Lebrija - Colombia. Rev univ. Ind. Santander [Internet] 2012. [Consultado 03 de Marzo de 2017]; 44(3):13-23. Disponible en: http://www.scielo.org.co/scielo.php?script=sci_arttext\&pid $=\mathrm{S} 0121-08072012000300003$

20. Colombia aprende. Programa Nacional de Educación para la Sexualidad y Construcción de Ciudadanía [Internet] 2015. [Consultado 3 de Julio de 2017]. Disponible en: http://www.colombiaaprende.edu.co/ html/productos/1685/articles-172204_recurso_1.pdf

21. Acosta S, Ibáñez E, Alfonso A, Cifuentes L, Gamba S, Mojica C, Vargas V, Patiño E. Conductas de salud y factores de riesgo en la salud sexual y reproductiva de una población universitaria. NOVA - Publicación Científica En Ciencias Biómedicas. [Internet] 2014. [Consultado el 5 Julio de 2017]; 8(13):32-43. Disponible en: http://unicolmayor.edu.co/publicaciones/index.php/nova/article/ view/148/296

22. Hernando V, Álvarez MC, Arriola L, Arroyo S, BallesterE, et al. Conocimientos y uso de anticonceptivos en la población inmigrante latinoamericana en la Comunidad Autónoma de Madrid, Centro Nacional de Epidemiología. [Internet] 2015. [Consultado el 5 Julio 2017]; 13(4):37-48. Disponible en: https://www.researchgate.net/profile/Juan_De_Mata_Donado-Campos/publication/268323730_ Conocimientos_y_uso_de_anticonceptivos_en_la_poblacion_inmigrante_latinoamericana_en_ la_Comunidad_Autonoma_de_Madrid/links/552550ae0cf2e0b9c735dd3b/Conocimientos-y-usode-anticonceptivos-en-lapoblacion-inmigrante-latinoamericana-en-la-Comunidad-Autonoma-deMadrid.pdf

23. Camacho Rodríguez DE, Pabón Varela Y. Percepciones que afectan negativamente el uso del condón en universitarios de la Costa Caribe colombiana. Hacia promoc. salud. [Internet] 2014; [Consultado 3 Marzo de 2017]; 19(1):54-67. Disponible en http://www.scielo.org.co/pdf/hpsal/v19n1/v19n1a05.pdf

24. Langer A. El embarazo no deseado: impacto sobre la salud y la sociedad en América Latina y el Caribe. Rev Panam Salud Publica/Pan Am J Public Health. [Internet]2002. [Consultado 03 de Marzo de 2017]; 11(3):192-205. Disponible en: http://www.scielosp.org/pdf/rpsp/v11n3/9402.pdf

25. Encuesta Nacional de Demografía y Salud ENDS Colombia 2015. Profamilia. [Internet] 2015. [Consultado 2017 Mar 03] disponible en: http://profamilia.org.co/docs/Libro\%20RESUMEN\%20 EJECUTIVO.pdf

26. Rangel Flores YY, García Rangel M. Violencia en estudiantes universitarios en San Luis Potosí, México: Un estudio desde la perspectiva de género. Index Enferm. [Intenet] 2012. [Consultado 03 Marzo de 2017]21(3)141-144. Disponible en: http://scielo.isciii.es/scielo.php?script=sci_arttext\&pid $=\mathrm{S} 1132-12962012000200007$

27. Mohamed Mohand L, Herrera Torres L, Carracedo Cortiñas S. violencia de pareja en jóvenes estudiantes universitarios de diferente origen cultural. revista de educação e humanidades. [internet] 2014. [citado 
ISSN-PRINT

1794-9831

E-ISSN 2322-7028

Vol. 15 No. 1

Ene - Jun 2018

Cúcuta, Colombia
2017 Mar 03]; 5:223-236. Disponible en: https://dialnet.unirioja.es/servlet/articulo?codigo $=4733824$

28. Gómez López C, Murad R, Calderón M. Historias de violencia, roles, prácticas y discursos legitimadores. Violencia contra las mujeres en Colombia 2000-2010. [Internet] Colombia:2015. Disponible en: http://www. profamilia.org.co/docs/estudios/imagenes/7\%20-\%20VIOLENCIA\%20CONTRA\%20LAS\%20MUJERES\%20 EN\%20COLOMBIA.pdf

29. Cerezo Correa MP, Vergara Quintero MC, Nieto Murillo E, Cifuentes Aguirre OL, Parra Sánchez JH. Características de salud pública de estudiantes de una universidad privada de la ciudad de Manizales. [Internet] 2011. [Consultado 03 Marzo de 2017]; 16(1)73-86. Disponible en: http://www.scielo.org.co/pdf/hpsal/v16n1/ v16n1a05.pdf

30. Uribe Rodríguez AF, Orcasita Pineda LT. Conductas sexuales de riesgo en estudiantes universitarios de la ciudad de Cali-Colombia. Revista Virtual Universidad Católica del Norte. [Internet] 2009. [Consultado 09 de Marzo de 2017]; 27:1-31 Disponible en: http://revistavirtual.ucn.edu.co/index.php/RevistaUCN/article/view/99/196

31. Arias Castillo L, Vásquez Truissi ML, Dueñas EP, García LM, Tejada EL. Conocimientos, actitudes y prácticas relacionadas con las infecciones de transmisión sexual en estudiantes universitarios, revista centro de estudios en salud [Internet] 2010. [Consultado 09 de Marzo de 2017]; 1(12)43-49. Disponible en: http://revistas.udenar.edu. co/index.php/usalud/article/view/185/pdf

32. Rodríguez Cabrera A, Álvarez Vázquez L. Percepciones y comportamientos de riesgos en la vida sexual y reproductiva de los adolescentes, Rev Cubana Salud Pública. [Internet] 2006. [Consultado 09 de Marzo de 2017] 32(1). Disponible en: http://scielo.sld.cu/pdf/rcsp/v32n1/spu08106.pdf 